\title{
Infant Screening
}

National Cancer Institute

\section{Source}

National Cancer Institute. Infant Screening. NCI Thesaurus. Code C81177.

Diagnostic tests performed on infants to assess overall health and identify potential areas of concern. 